\title{
Multiple levels of spatial organization: World Graphs and spatial difference learning
}

Adoptive Behavior 20(4) 287-303

(c) The Auchor(s) 2012

Reprints and permissions: sagepub.co.uk/journalsPermissions.nav DOI: 10.1177/1059712312449545 adb.sagepub.com (SSAGE

\author{
Michael A Arbib ${ }^{1,2,3}$ and James J Bonaiuto ${ }^{2,3}$
}

\begin{abstract}
It is often suggested that the place cells of the hippocampus (and more recently, the grid cells of the entorhinal cortex) furnish a cognitive map. However, this can only be part of the story: (I) the "hippocampal chart" provided by place cells and grid cells differs radically when a rat is placed in different environments and so a higher level organization is needed to link these charts into an overall cognitive map of the rat's world; and (2) even without a hippocampus a rat can exploit much of the spatial structure of its world. The World Graph (WG) model addressed the former problem, whereas the Taxon Affordance Model (TAM) was developed to address the latter, with the two models being integrated to form the TAM-WG model. Here we provide a new version strengthened in three ways: (1) we relate the TAM model to explicit ideas of executability and desirability; (2) we show how temporal difference learning elegantly supplies "spatial difference learning" to resolve the debate between the local hypothesis and the non-local hypothesis for node selection in the original WG model; and (3) we analyze an explicit example of how the "locometric map" provided by grid cells and place cells can complement the high-level cognitive map given by the WG, demonstrating the importance of navigation algorithms that integrate across multiple levels of spatial organization.
\end{abstract}

\section{Keywords}

Hippocampus, navigation, reinforcement learning, affordances, cognitive map

\section{Introduction: the World Graph and the experience of space}

Ever since the publication of The Hippocampus as a Cognitive Map (O'Keefe \& Nadel, 1978), it has indeed been fashionable to think of the hippocampus as providing a map. We argue instead that it provides just one component of a map, and offer a comprehensive computational model of multiple maps in the brain and the neural mechanisms that exploit them. First, we offer a working definition of a spatial map that grounds our discussion of how the hippocampus plus other systems provide a variety of spatial maps.

Definition 1: A map $\mathbf{M}$ for a user $\mathbf{U}$ of a space $\mathbf{S}$ is a representation of a limited "sample" of $S$ such that:

(1) $U$ can find in $M$ a representation $M(A)$ of $U$ 's current location $A$

(2) $U$ can find in $M$ a representation $M(B)$ of $U$ 's desired location $B$

(3) $U$ can find in $M$ a path $P M(A, B)$ from $M(A)$ to $M(B)$
(4) $U$ can transform $P M(A, B)$ into a path $P S(A, B)$ from $A$ to $B$ in $S$.

Note that this definition (1) makes explicit the user $\mathrm{U}$ of the map, and then (2) makes clear that $\mathrm{U}$ must be able to use the map. This definition thus depends as much on U's capabilities as it does on M's properties. In the case of a paper or digital map, the map is external to the brain of the user. However, when we talk of "maps in the brain", we normally refer to the encoding of $\mathrm{M}$ in the brain of $U$ while assuming (but often failing to make explicit) that mechanisms exist within U's

'Computer Science Department, University of Southern California, Los Angeles, CA, USA

${ }^{2}$ Neuroscience Graduate Program, University of Southern California, Los Angeles, CA, USA

${ }^{3}$ USC Brain Project, University of Southern California, Los Angeles, CA, USA

\section{Corresponding author:}

James Bonaiuto, California Institute of Technology, 1200 E California Blvd, M/C 216-76, Pasadena, CA 91225 , USA

Email: bonaiuto@caltech.edu 
brain to use the map in the way defined above. In this case, we may paraphrase the conditions as:

(1) Perception of U's current location;

(2) Goal-setting: establishing criteria for where $U$ wants to go;

(3) Planning a route to a goal;

(4) Execution: converting the plan into a course of action.

The formulation of (2) makes it clear that the goal may not be a specific place so much as a place where certain needs can be met (e.g., a good restaurant).

As the animal moves in different ways, or makes use of different sensory cues in guiding its movement, its spatial behavior exploits a variety of different representations in its brain. We can distinguish diverse "maps" in each animal's brain (though most such maps only satisfy criterion (1) and/or (2) in Definition 1): representations of oculomotor space, representations that guide locomotion, representations that guide reaching, and many more (Arbib, 1997b; Colby, 1998). The brain's multiple maps gain their coherence not by their subservience to some overarching mathematical definition of space but with respect to a repertoire of actions.

Our navigation through the world proceeds at multiple levels of spatial organization. If I want to go to London, my route may take me to my local airport, then by plane to Heathrow, and by the tube to Piccadilly. My knowledge of the world needed to support such high-level navigation, according to our model, can be represented computationally by a World Graph (WG; Arbib \& Lieblich, 1977; Lieblich \& Arbib, 1982), each node of which corresponds to a recognizably distinct location in the world, with each edge representing a route between two locations and/or the actions necessary to trace that route. A similar representation holds for a subway map. The WG model gives an account not only of how a high-level cognitive map could guide behavior but also of how such a map could be acquired through experience. Moreover, it emphasized the way in which the "read out" of the map would depend strongly on the motivational state of the animal.

Although a subway map helps us navigate a city, it has little metric structure. Yet, just as when we descend from the train at a station we re-enter the world of locomotion, so the subway map must be complemented by a space that is "more metric" in nature. We coin the term locometric for the way an animal measures the world in terms of locomotory actions (e.g., how many steps taken) or perceived measures of such actions (e.g., the visual effect of an action such as the achievement of a goal). Interestingly, the bee's "odometer" is based on integration of optic flow, rather than energy expended or a count of wing beats (Srinivasan, Zhang, Altwein, \& Tautz, 2000). The notion of locometric space is particularly relevant to the notion of path integration, the ability of a wandering animal to keep track of the location of its home base relative to its current position, a capability that has been related to dynamic remapping of the hippocampal map (Guazzelli et al., 2001). This brings us close to the hippocampal "place system", thought to encode the location of the animal in a particular environment (O'Keefe \& Dostrovsky, 1971). In a radial maze, for example, several place fields may cover the full length of each arm of the maze, yet the corresponding WG might have nodes only for the center of the maze and the far end of each arm.

From now on, we will refer to the modeled animal as a "rat", while stressing that the model may account for aspects of navigational behavior in many species, including humans. What we now want to re-emphasize (a point made by Lieblich \& Arbib, 1982) is that the hippocampus does not encode a global map. When a rat is placed in different environments, a place cell will code places in each environment but there will be no apparent relation between its roles in the two maps. Let us call each such map a "hippocampal chart". Thus, in what follows, we suggest that different subgraphs of an animal or human's WG may correspond to different hippocampal charts. If a rat lives in a particular cage, the hippocampal chart may give a detailed locometric representation of the space (which can extend to 3D when jumping and climbing are included in the set of locomotor actions), while the nodes of the WG may encode a limited set of significant locations, such as those of the drinking tube, feeder, nest and running wheel.

In accord with WG theory, the model contains a set $d_{1}, d_{2}, \ldots, d_{k}$ of drives, each of which has value $d_{i}(t)$ at time $t$. Each value $d$ is constrained to lie between values $d_{\max }$ and $d_{\min }$. Drives can be appetitive or aversive, where appetitive drives spontaneously increase to $d_{\max }$, while aversive drives are reduced to $d_{\min }$, each according to a factor $\alpha_{d}$. Drives are additionally increased if an incentive stimulus $S T(d, t)$ (such as the sight of food for hunger; the sight of a predator for fear) increases or decreases, the $\Delta S T(d, t)$ in the next equations, and are reduced by a factor $c(d, t)$ given the appropriate conditions (i.e., ingestion of food reduces hunger, Figure 1; while escape may reduce fear). Note that hunger and thirst are reduced by behavior at a particular site, whereas fear is reduced by escape - "freezing" probably does not reduce fear, but rather persists until the fearful stimulus passes. Section 2, Materials and Methods, gives the necessary equations for modeling.

Different goal-setting criteria support different goals. Planning a hike, we may want a contour map; going for a drive, we want a road map. Thus, the National Geospatial-Intelligence Agency (www.nga. mil) provides digital maps on computers, which have geospatial information in layers. Two of the many layers that NGA provides are: (1) buildings such as houses, schools, shopping malls, museums, stadiums, 


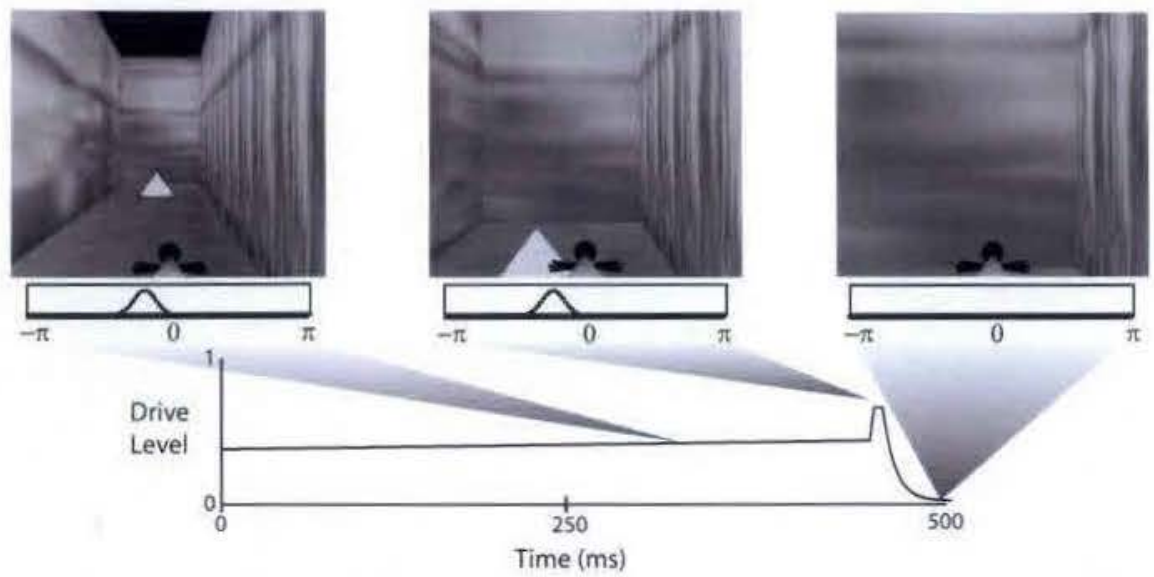

Figure 1. Top: egocentric view as the simulated rat approaches a piece of food. Middle: activity of the incentive-sensing neurons. Bottom: hunger level as the food is approached and as it is consumed. The figure shows how drive levels change both spontaneously and in the presence of incentives, then decrease as food-taking reduces hunger.

government buildings, and businesses (knowing the locations of buildings helps people travel and find places to, e.g., take shelter) and (2) navigation aids such as lighthouses, beacons, buoys, light towers, markers, and bells, showing the heights and light characteristics on navigation charts enables sailors and pilots can match what they see outdoors to what they see on the charts. This latter comment is particularly interesting because it relates what the user sees (the egocentric view) to the map representation (the allocentric view), tying back to item (1) of our Definition.

In the WG model, we have the equivalent of one overlay for each drive $d_{i}$, but with the extra capacity that it can be updated over time by reinforcement learning. $R\left(d_{i}, x, t\right)$ is the value of the $d_{i}$ overlay at time $t$ for position $x$ on the map. The motivational system provides the rat (and this model) with a set of low-level goals to pursue. Each goal is tied to the reduction of a specific drive. For example, the goal of eating food is associated with the hunger drive. This appetitive drive spontaneously increases and the sight of a food-related incentive stimulus ramps up the increase of that drive. The hypothalamus contains functionally related subsystems for regulation of eating, drinking, sex, and temperature (Swanson \& Mogenson, 1981), and we follow Swanson $(2000,2005)$ in postulating that it influences behavior through its connections with the basal ganglia and prefrontal cortex. In this model, the output of the hypothalamus is used to map reward signals onto utility for each drive, and this utility will serve as the reinforcement signal $r(d, t)$ for the learning of information relevant to a particular drive $d$. The idea behind this is that if the rat is not very hungry, a reduction in the hunger drive has lower utility than when it is extremely hungry. It has been suggested that motivations transform raw reward signals into utility based on internal state (Goldman, 2006). The lateral hypothalamus contains subregions that seem to encode internal state variables, and produces orexins (hypocretins), which are regulated by metabolic cues (Sakurai, 2006). Orexin-containing hypothalamic neurons project to dopamine-releasing neurons in the ventral tegmental area (VTA), which is implicated in reward and reinforcement learning (Vittoz \& Berridge, 2006). Orexin B facilitates glutamatergic activation of VTA neurons by increasing presynaptic transmitter release and potentiating postsynaptic NMDA receptors (Borgland, Storm, \& Bonci, 2008), suggesting that internal state variables modulate the gain of reward signals.

The use of these drive-related "overlays" makes our setup a model of "motivational learning of spatial behavior" in the sense that what is important in the cognitive map, and thus how it is learned, depends very much on the motivational state of the animal.

The basic notion of an affordance is that the world may be perceived in terms of perceptual cues, which guide action as much as in terms of the identity of objects. Thus, in landing a plane one certainly needs to recognize the runway, but the crucial affordance for action is the optic flow, which tells the pilot the time until contact with the ground (Gibson, 1955). The shape of an object provides affordances for grasping (Arbib, 1997a), and, in navigating a maze, the recognition of walls and alleys as such may be secondary to the recognition of affordances for locomotion. Based on the activity of rat posterior parietal cortex before movements in a maze (McNaughton, Leonard, \& Chen, 1989; McNaughton et al., 1994), the Taxon Affordance Model (TAM; Guazzelli, Corbacho, Bota, \& Arbib, 1998) hypothesizes that the parietal cortex encodes, among other things, possible directions of locomotion in an egocentric reference frame.

In many cases, locomotion may be based solely on the affordances in one's immediate environment. 
Indeed, TAM explores the unmediated response to current affordances based on the desirability (expectation of current and future reinforcement) of actions whose executability is signaled by those affordances. A locomotive affordance is a direction that permits locomotion in that direction, which interacts with landmarks and learned reinforcement in action selection. Therefore, the output of the model is a series of waypoints for goal-directed locomotion and control of actuators is left to low-level motor controllers. These affordances, for movement in directions from $-\pi$ to $\pi$, are represented as population codes by directionselective neurons in the Parietal Cortex.

The architecture of the TAM model is shown in Figure 2 (reconfigured from the original). The visual cortex provides an egocentric view of the local environment to the Parietal Cortex module and signals detected incentives to the Hypothalamus (although a more complete model with senses such as olfaction would have multisensory incentive inputs to the Hypothalamus). The Somatosensory Cortex detects adjacent obstacles whether they are visible or not and projects this information to the Parietal Cortex module. The Parietal Cortex module combines information from the egocentric view and somatosensation to provide the Premotor Cortex/Actor with an executability signal for possible actions. The Premotor Cortex/Actor module multiplicatively combines the egocentric executability and movement direction desirability signals to form priorities for the next movement direction, selecting the one with the highest priority.
An important result of the TAM model was to show that it could reproduce the behavior of a rat with lesioned fornix whose behavior was thus uninfluenced by the hippocampus (O'Keefe, 1983). Recent studies suggest that the rat posterior parietal cortex encodes directions in a route-based reference frame (Nitz, 2009) and integrates the current orientation with potential spatial goals (Calton \& Taube, 2009) by elaborating the hippocampal map using proximal landmarks (Save, Paz-Villagran, Alexinsky, \& Poucet, 2005). Such results can best be understood by placing route selection within the context provided by the WG theory, complementing the scope of the TAM model.

Going beyond TAM, the original TAM-WG model added a network composed of feature detectors whose output is used to drive hippocampal encoding of the current location using place cells. This is similar to the Simultaneous Localization And Mapping (SLAM) problem in robotics. We now emphasize an important distinction: place cells may cover the current area of exploration, but WG-nodes only encode places that have special significance, such as providing reinforcement or requiring a marked change in an ongoing course of action. Thus the current location might be recognized as one represented by an existing node of the WG, as a place of no special interest, or as an as-yet-unrepresented place of interest. In the last case, the WG model will add a new node to WG, together with an edge linking it to the node for the last visited place of interest. Milford et al. (Milford, Wyet, \& Prasser; 2004; Milford, Schulz, Prasser, Wyeth, \&

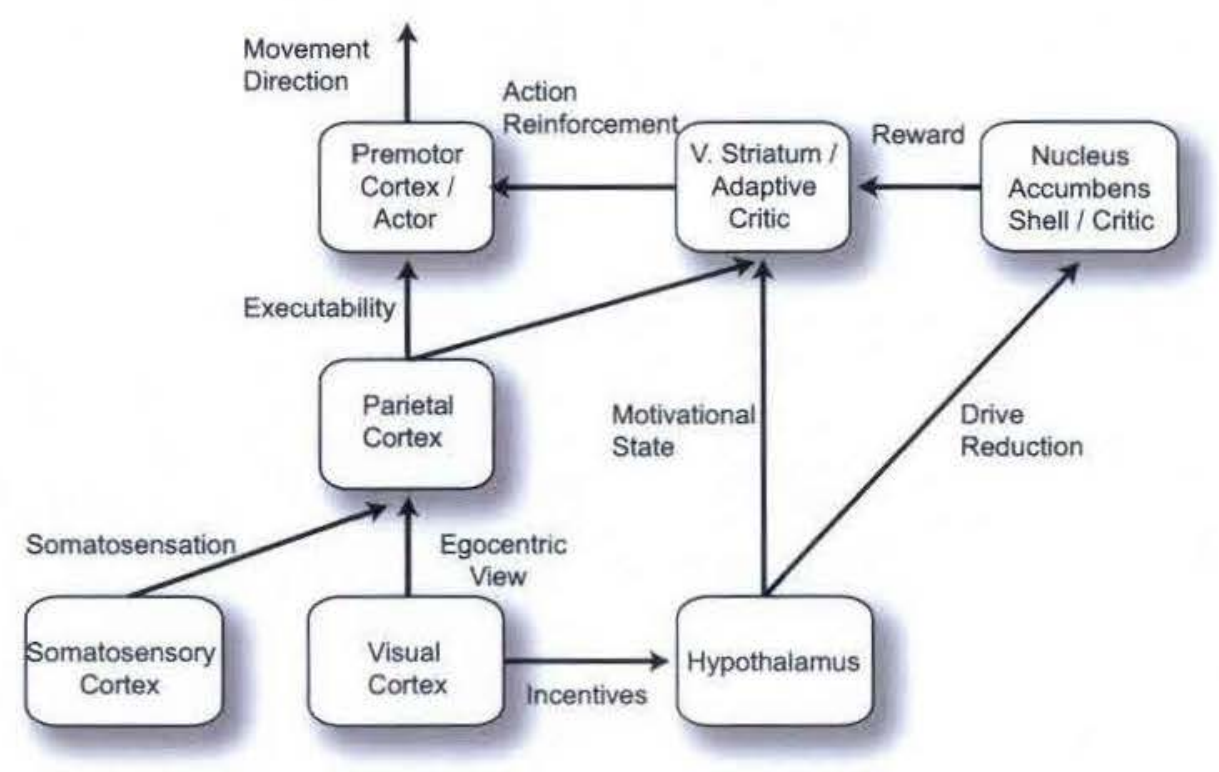

Figure 2. Taxon Affordance Model (TAM) components. The premotor cortex combines an executability signal from parietal cortex with a desirability (action reinforcement) signal based on motivational drives to select the next movement direction. (Adapted from Guazzelli et al., 1998.) 
Wiles 2007; Milford \& Wyeth, 2010) developed a SLAM algorithm, RatSLAM, based on a model of the rat hippocampal system. We therefore do not model the formation of the locometric map here and simply provide the WG module with noisy input signaling the current location of the rat. When a place of interest is reached for which no node exists in the graph, a new node is added, using a working memory trace of the last node and an efference copy of motor output and or associated perceptual experience to label a directed edge with orientation information to the new node. In the examples used below, a junction or end of corridor is a place of interest (because of the possible or forced change of direction), as is a location where reinforcement is received.

Both TAM and WG uses a form of temporal difference reinforcement that we term "spatial difference learning" to learn expectations of future reward. The key differences are that in the WG module the expectations are associated with node/edge rather than state action pairs, the action space is the direction of movement in an allocentric reference frame, and the discounting between subsequent states depends on the spatial distance between them.

We have recently introduced a model of action selection called Augmented Competitive Queuing (ACQ: Bonaiuto \& Arbib, 2010). ACQ selects which action to perform on the basis of what we term desirability (expected reward) and executibility (probability of success). Executability is based on the perceived affordances for actions as well as the effort or cost of an action, whereas desirability is solely related to expectation of reward. These signals are multiplied to yield an action priority signal that is then input to a winnertake-all (WTA) process, which chooses the action with the highest priority to perform. We adapt the original TAM-WG model using this framework. In TAM-WG, action desirability is determined by reinforcement learning and action executibility is determined by perceived affordances. The two influences are combined to select the next action and control or interrupt currently ongoing actions.

\section{Materials and methods}

The present paper describes a simulation model, which has been implemented using the Java-based version of the NSL Neural Simulation Language (Weitzenfeld, Arbib, \& Alexander, 2002; http://neuroinformatics.usc.edu/mediawiki/index.php/NSL) interfaced with Java3D for visualization. The present section describes the framework and mathematical equations for the model; the simulation results then appear in the Results section. The time step in these equations corresponds to the time in which it takes a rat to move one or two steps in locometric space - about $500 \mathrm{~ms}$. It may therefore take several time steps to traverse a route between two landmarks in a maze.

\section{I Motivational system}

We now present the equations that underlie the dynamics shown in Figure 1, modifying those of Arbib and Lieblich (1977). Given the value of a drive $d(t)$ at time $t$, the value at time $t+1$ is given by:

$$
\begin{aligned}
d(t+1)= & d(t)+\alpha_{d}\left(d_{\max }-d(t)\right) \\
& -c(d, t)\left|d(t)-d_{\min }\right|+\Delta S T(d, t)\left|d_{\max }-d(t)\right|
\end{aligned}
$$

for an appetitive drive, and

$$
\begin{aligned}
d(t+1)= & d(t)-\alpha_{d}\left(d(t)-d_{\min }\right) \\
& -c(d, t)\left|d_{\max }-d(t)\right|+\Delta S T(d, t)\left|d_{\max }-d(t)\right|
\end{aligned}
$$

for an aversive drive. For an appetitive drive like hunger, the change in $d$ combines a spontaneous increase towards the maximum value, a reduction in $d$ caused by appropriate behavior, and the increase in $d$ caused by an increase in current incentives. By contrast, the value of an aversive drive like fear will spontaneously decrease towards its minimum in the absence of other effects. Drive reduction, $c(d, t)$ is only positive for appetitive drives, but could be negative for aversive drives (i.e., $c($ fear,$t)<0$ ), thus increasing the fear drive, making one more fearful.

Several components of the model make use of the normalized drive level, $\hat{d}(t)$, which is given by:

$$
\hat{d}(t)=\frac{d(t)-d_{\min }}{d_{\max }-d_{\min }}
$$

We have suggested that internal state variables modulate the gain of reward signals. We model this effect by postulating that the utility $r(d, t)$ of the rate of drive reduction $c(d, t)$ depends on the current normalized value of that drive $d(t)$ :

$$
r(d, t)=\hat{d}(t) c(d, t)
$$

The original WG model (Arbib \& Lieblich, 1977) attached to each node $x$ of $W G(t)$ the vector

$$
\left[R\left(d_{1}, x, t\right), \ldots, R\left(d_{k}, x, t\right)\right]
$$

where $R\left(d_{i}, x, t\right)$ is the animal's internal representation, based on experience prior to $t$, of the extent to which substrates at the place represented by node $x$ ("at node $x$ ", for short) serve to reduce drive $d_{i}$, or at least provide reinforcement related to drive $d_{i}$. If the animal is at node $x$ at time $t$, then $R(d, x, t)$ changes to $R(d, x, t+l)$ in a manner that depends on the utility $r(d, t)$. Before describing how the $R$-values are updated, we first note 
two different hypotheses on how the animal chooses the next action:

- The Dominant Node Hypothesis: the animal goes to a node most likely to reduce its dominant drive.

- The Competing Nodes Hypothesis: the animal goes to a node with the greatest expected reduction of all drives.

In the former case, a hungry animal might go to the node that may provide the most food, irrespective of its utility with respect to other drives. In the latter case, the appeal of food and water at a node would be combined, and this would in turn be reduced by fear associated with the node. We will later review experimental data and show that its successful modeling employs the competing nodes hypothesis.

But how do these expected reinforcements change? The original model was developed many years before the invention of the mathematical theory of temporal difference learning (Sutton, 1984; Sutton \& Barto, 1998). The key idea of the latter is that reinforcement may be intermittent, so that when we act it will often be not on the basis of any expectation of immediate reinforcement, but rather on the basis of our expectation that our current choice will lead to further opportunities whose cumulative effect will be to yield near optimal positive reinforcement. The WG model anticipated the problem, but lacked the systematic answer that temporal difference learning provides (see the details below). Arbib and Lieblich (1977) considered two alternatives:

- The Local Hypothesis: the weights assigned to node $x$ in the node selection process depend only on the $R(d, x, t)$ for that $x$.

- The Non-local Hypothesis: the weights assigned a node $x$ in the node selection process depend not only on the $R(d, x, t)$ for that $x$, but also on the $R\left(d, x^{\prime}, t\right)$ for nodes $x^{\prime}$ reachable on paths through $x$.

In our current work, consistent with the available biology, we adopt the non-local hypothesis - but now with the power of "spatial difference learning", which is just a fancy way of saying that we apply temporal difference learning to the selection among the edges available at each node of the WG, so that the each node comes to be associated with a better and better estimate of the total discounted reinforcement to be expected if one chooses the optimal path emanating from $x$.

\subsection{TAM - the Taxon Affordance Model}

The TAM accounts for how the rat acts in recognizable situations such as "in corridor, with branches in directions $(\mathrm{a}, \mathrm{b}, \mathrm{c} . .$.$) visible" that do not involve a map that$ encodes where this situation arises. Taking such a map into account will be the task of the WG model to be described in Section 2.3. So whereas in the previous section the state, $x$, was a node in the WG, in TAM $x$ refers to a recognizable situation, which could possibly encode the same location of the maze as different states if the angle of direction is different.

Consumption of food or water provides the coefficient of reduction, $c(d, t)$, of the appropriate drive, while the utility $r(d, t)$, obtained by scaling $c(d, t)$ by the current normalized value of the drive, is used as a reinforcement signal. TAM uses reinforcement learning to learn action desirability - expectations of future reward for state/action pairs. The typical convention for temporal difference learning with an actor-critic architecture uses the output $P(t)$ of an adaptive critic to predict the discounted sum of future reward (Sutton \& Barto, 1998). The prediction is used as reinforcement for the actor, and the error in this prediction, the temporal difference error, $\hat{r}(t)$ is used to train the adaptive critic.

$$
\hat{r}(t)=r(t)+\gamma P(t+1)-P(t)
$$

where $\gamma$ is the discount factor.

The output of the critic represents a prediction of discounted sum of future utility achievable from state $x$. In the current simulations, the state space consists of combinations of sensory input that encode recognizable situations. The action space is the direction of movement in an egocentric reference frame (Figure 3 ).

The temporal difference error is then determined by the total utility, $\sum r(d, t)$, and the temporal difference in predictor output weighted by the sum of the normalized magnitude of all the drives:

$$
\begin{aligned}
\hat{r}_{\text {TAM }}(t)= & \sum_{d} r(d, t) \\
& +\sum_{d} \hat{d}(t)\left[\gamma P_{\text {TAM }}(x, t+1)-P_{\text {TAM }}(x, t)\right]
\end{aligned}
$$

The prediction of future utility, $P_{T A M}(x, t+1)$ is discounted by a factor, $\gamma$.

The critic then updates its predictions for that state:

$$
\Delta P_{\text {TAM }}(x, t)=\beta_{\text {TAM }} \hat{r}_{\text {TAM }}(t) e_{\text {TAM }}(x, t)
$$

where $\beta_{T A M}$ is the learning rate, and $e_{T A M}(x, \mathrm{t})$ is an eligibility trace (the subscript distinguishes these values from those employed in the WG model). Eligibility traces are commonly used in actor/critic reinforcement learning in order to assign credit to the appropriate state/action pair for the delayed reward from the adaptive critic (Singh \& Sutton, 1996). An analysis of midbrain dopaminergic cell activity suggests that temporal difference models require long-lasting eligibility traces to replicate their firing patterns during conditioning (Pan, Schmidt, Wickens, \& Hyland, 2005). The eligibility signal reaches its maximum for a particular 


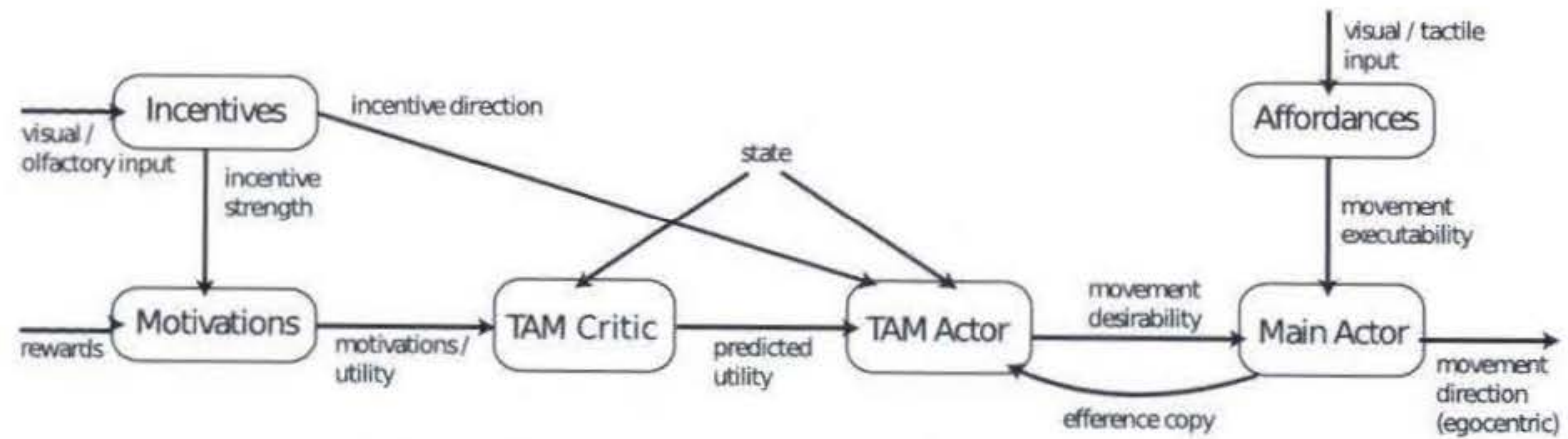

Figure 3. A functional view of the Taxon Affordance Model (TAM) module. Compare the functional view of the World Graph (WG) module provided in Figure 4. Figure 6 shows how the two modules are integrated to provide the overall TAM-WG model.

state immediately after encountering it and thereafter decays to 0 .

The actor uses the prediction output of the adaptive critic to update its policy $\mathbf{W}$, which is a mapping from state-space to action-space. In this model, the policy is implemented as a vector of values for each state that encodes the preferred direction of movement in that state. The actor updates its policy so that it chooses the action $a$ leading to a new state $a[x]$ that maximizes $P_{\text {TAM }}(a[x], t+1)$.

This is accomplished by using the adaptive critic's prediction for the current state to reinforce the policy for the last state and the action performed in it:

$$
\begin{aligned}
& \Delta \mathbf{W}_{\text {TAM }}(x, t+1)=\eta_{\text {TAM }} \\
& P_{\text {TAM }}(a[x], t+1) e_{\text {TAM }}(x, t+1) \mathbf{z}(t+1)
\end{aligned}
$$

where $\mathbf{z}(t+1)$ is a decaying efference copy of the output of the actor in state $x$ at time $t$. The policy is then normalized according to its maximal value and used to generate an action desirability signal, $\mathbf{A D}(x, t)$, when in state $x$. This signal is a generated by computing a coarse population code over the center of mass of the vector, resulting from the product of the sum of the normalized drive levels and the corresponding policy for state $x$ :

$$
\sum_{d} \hat{d}(t) \mathbf{W}_{\text {TAM }}(x, t)
$$

The actor module performs a WTA process over the noisy integration of the motivation, incentive, affordance, and desirability signals. The total desirability is given by the sum of the action desirability, AD, and a coarse population code of the location of the incentive stimuli, ST, over all drives. The desirability is gated by the output of the Parietal Cortex, E, representing movement executability. This ensures that only possible directions of movement are considered and with a competitive strength proportional to their executability and desirability. The entire input to the WTA process, $\mathbf{I}_{i n}$, is a vector combining the executability, incentive stimuli, and action desirability signals:

$$
\mathbf{I}_{i n}(t)=\mathbf{E}(t)\left[\mathbf{A D}(d, x, t)+\sum_{d} \mathbf{S T}(d, t)+\varepsilon\right]
$$

where $\varepsilon$ is a noise term.

All populations in these simulations contained 200 neurons with selectivity for movement in directions from $-\pi$ to $\pi$. A dynamic neural field (DNF; Amari \& Arbib, 1977; Erlhagen \& Schoner, 2002) is used to implement the WTA process, resulting in a population code over movement direction centered on the cell with the highest mean input. When the maximum firing rate of this field reaches a threshold, this population code is decoded using the center-of-mass technique $(\mathrm{Wu}$, Amari, \& Nakahara, 2002) and the resulting direction along with the distance to the selected affordance is used to set the target location for the motor controller. Once a target location is set, the motor controller rotates the model avatar until it is facing that direction and advances toward the target. This process can be interrupted if the DNF sets a different target location before the current target is reached. In this manner, the continuous computation of affordances and desirability can affect and interrupt an ongoing action.

\subsection{The World Graph model}

The WG starts from a single node representing the current location. In the WG model, the state, $x$, is determined by the node. In a novel environment, surrounding landmarks that do not correspond to existing nodes are added as new nodes to the graph along with edges connecting to them from the current node if an affordance is perceived in that direction.

Similarly to the TAM critics, the WG critics compute the error in their predictions. However, in the WG model, we use multiple critics with output $P_{W G}(d, x, t)$, one for each dimension $d$ of the motivational state. The temporal difference error for a particular drive is then determined by the utility, $r(d, t)$, and the temporal difference in predictor output weighted by the normalized 


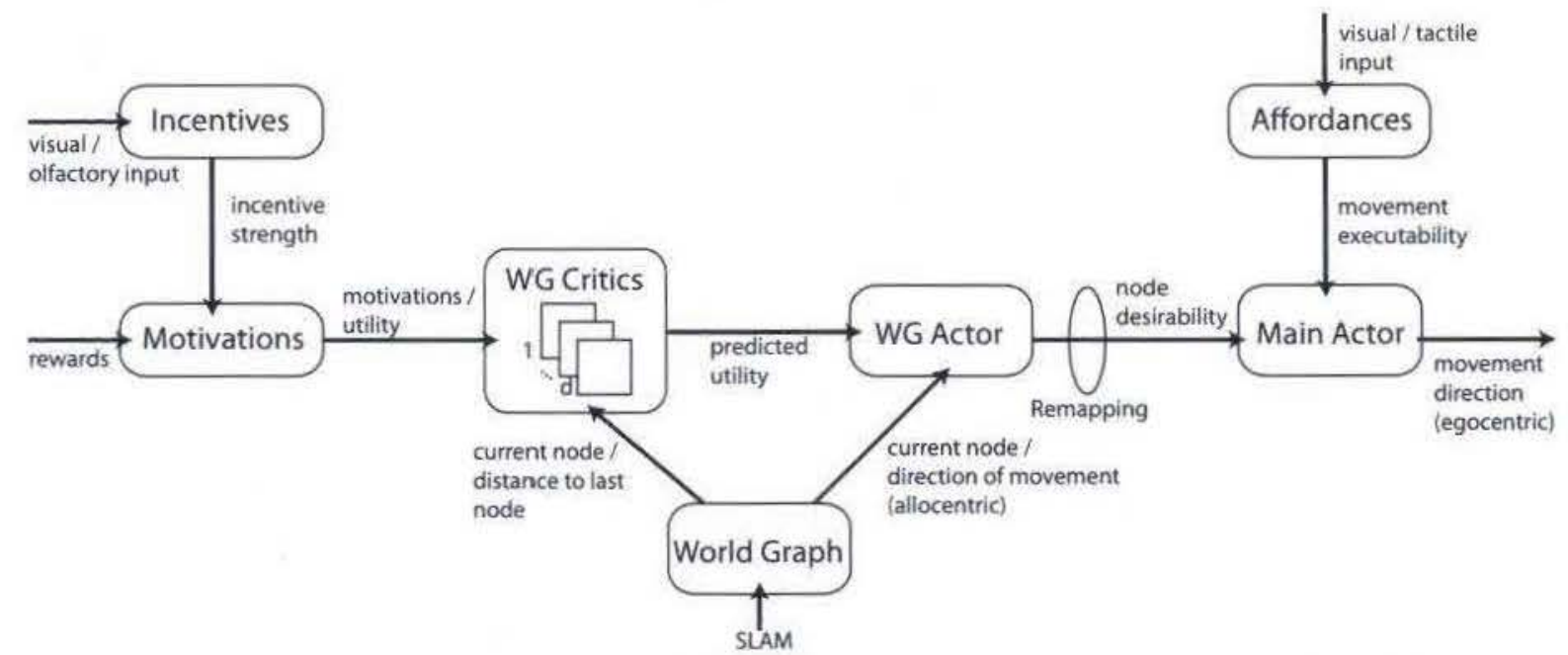

Figure 4. A functional view of the World Graph (WG) module. Figure 6 shows how it is integrated with the Taxon Affordance Model (TAM) to provide the overall TAM-WG model.

magnitude of the drive: each WG critic then computes the error in its prediction, $\hat{r}_{W G}(d, t)$ :

$$
\begin{aligned}
\hat{r}_{W G}(d, t)= & r(d, t)+\hat{d}(t)\left[\gamma_{d}^{\ell} P_{W G}(d, x, t+1)\right. \\
& \left.-P_{W G}(d, x, t)\right]
\end{aligned}
$$

where $x$ denotes a node in the WG in this case, $\gamma_{d}$ is a drive-specific discount factor, and $\ell$ is the perceived spatial distance traversed between the last node visited and the current one (Figure 4). The spatial difference error is used to update a prediction of the discounted reward achievable when in each node.

$$
\Delta P_{W G}(d, x, t)=\beta_{W G} \hat{r}_{W G}(d, t) e_{W G}(x, t)
$$

As with the $e_{T A M}$ signal, $e_{W G}$ is a decaying eligibility trace encoding the node most recently visited.

The actor uses the WG critic's prediction to learn a policy encoding preferred actions in each node for each drive:

$$
\begin{aligned}
& \Delta \mathbf{W}_{W G}(d, x, t+1)=\hat{d}(t) \eta_{W G} P_{W G}(d, a[x], t+1) \\
& e_{W G}(x, t+1) \mathbf{w}(t+1)
\end{aligned}
$$

which is then normalized according to its maximal value. As in the TAM component, the action space is movement direction, but in an allocentric reference frame. The action eligibility signal in this case, w, is therefore a decaying population code of the allocentric direction of the last movement. This policy is used to set a node desirability bias, $\mathbf{N D}(d, x, t)$, which encodes preferred movement direction in a coarse population code over the allocentric directions of nodes adjacent to the node $x$. Similar to the action desirability bias signal, the node desirability bias is weighted by the normalized drive level:

$$
\mathbf{N D}(d, x, t)=\hat{d}(t) e^{-\frac{1}{d}} \mathbf{W}_{W G}(d, x, t)
$$

The policy update is weighted by the current drive level so that hunger policies, for example, are only updated when the hunger drive level is significant. Unlike the action desirability signal, the node desirability is discounted by the perceived spatial distance to the next node, according to a drive-specific discount factor, $\kappa_{d}$. Depending on the value of $\kappa_{d}$, this discounting ensures that the influence of an adjacent node value is scaled according to its distance.

\subsection{Integrated model}

The combined model exerts influences from both the TAM and WG models on the Premotor Cortex/Actor module for action selection (see Figure 5 for an anatomical view and Figure 6 for a functional view).

In the Figure 5 view of the model, extending the TAM view of Figure 2, the visual cortex additionally provides an egocentric view of the local environment to the Hippocampus/SLAM module. This module determines the current allocentric orientation and position on the basis of the current egocentric view and provides this information to the PFC/WG module. The Hypothalamus provides the PFC/WG with the current motivational state. The Ventral Striatum/Adaptive Critic combines the motivational state, reward, and node information to perform spatial difference learning on the nodes in the WG, providing the PFC/WG module with a reinforcement signal. The PFC/WG module determines the current node on the basis of the orientation/position signal from the Hippocampus/SLAM module and provides the Premotor Cortex/Actor module with a coarse egocentric desirability bias on the basis of the current motivational state. Note that the 


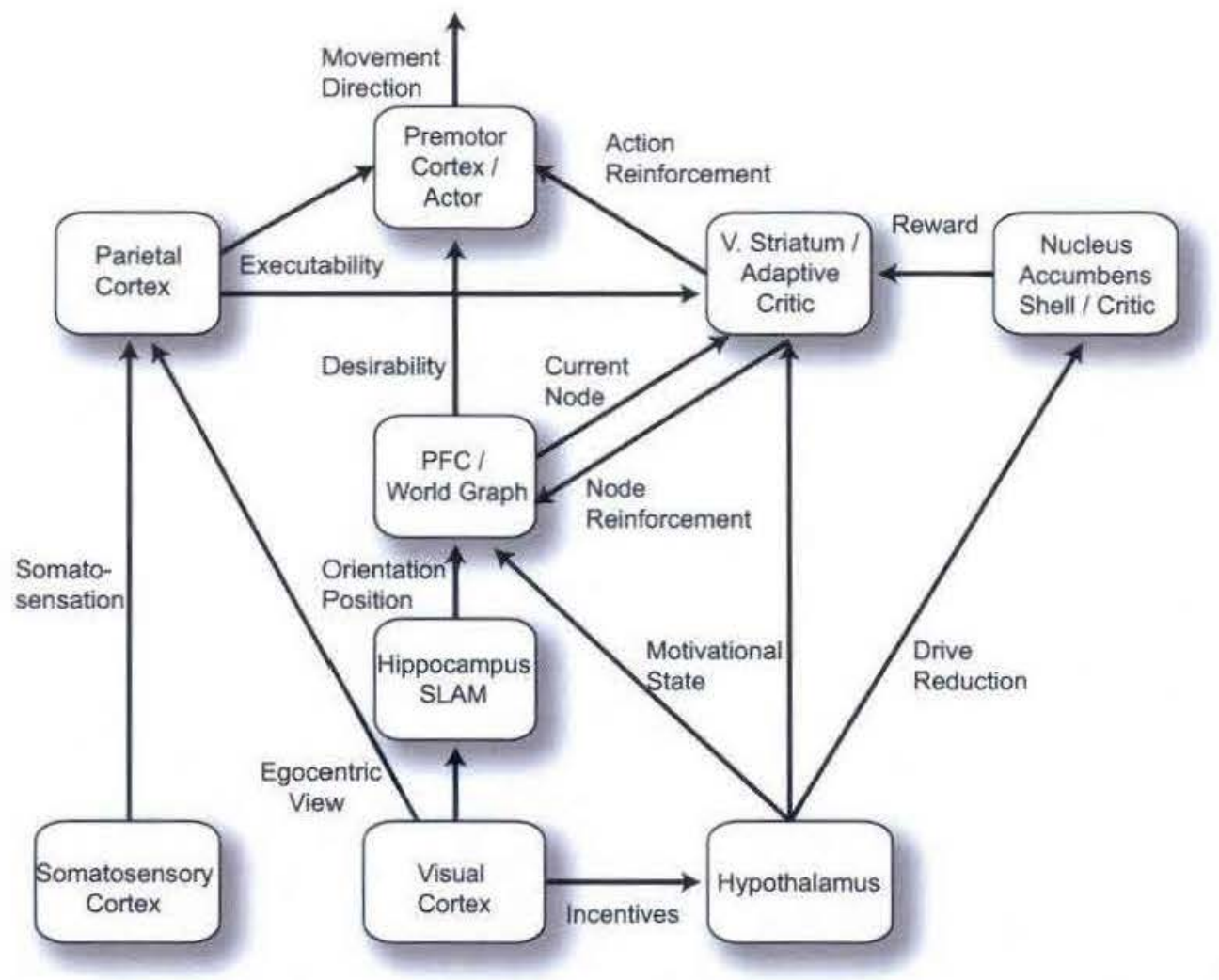

Figure 5. An anatomical view of the integrated Taxon Affordance Model-World Graph (TAM-WG) model. The PFCMorld Graph module now exerts an additional influence on the Premotor Cortex/Actor module.

WG output is in an allocentric reference frame, and must therefore be converted to an egocentric reference frame before combination with the output of the TAM and Parietal Cortex components. We use the notation $\Phi(\mathbf{x}, \theta)$ to represent a function that shifts a population code of allocentric orientation, $\mathbf{x}$, according to the rat's orientation, $\theta$, to yield a population code of egocentric orientation. We use a procedural implementation of this process in these simulations, but note that a similar mechanism, dynamic remapping, and its neural

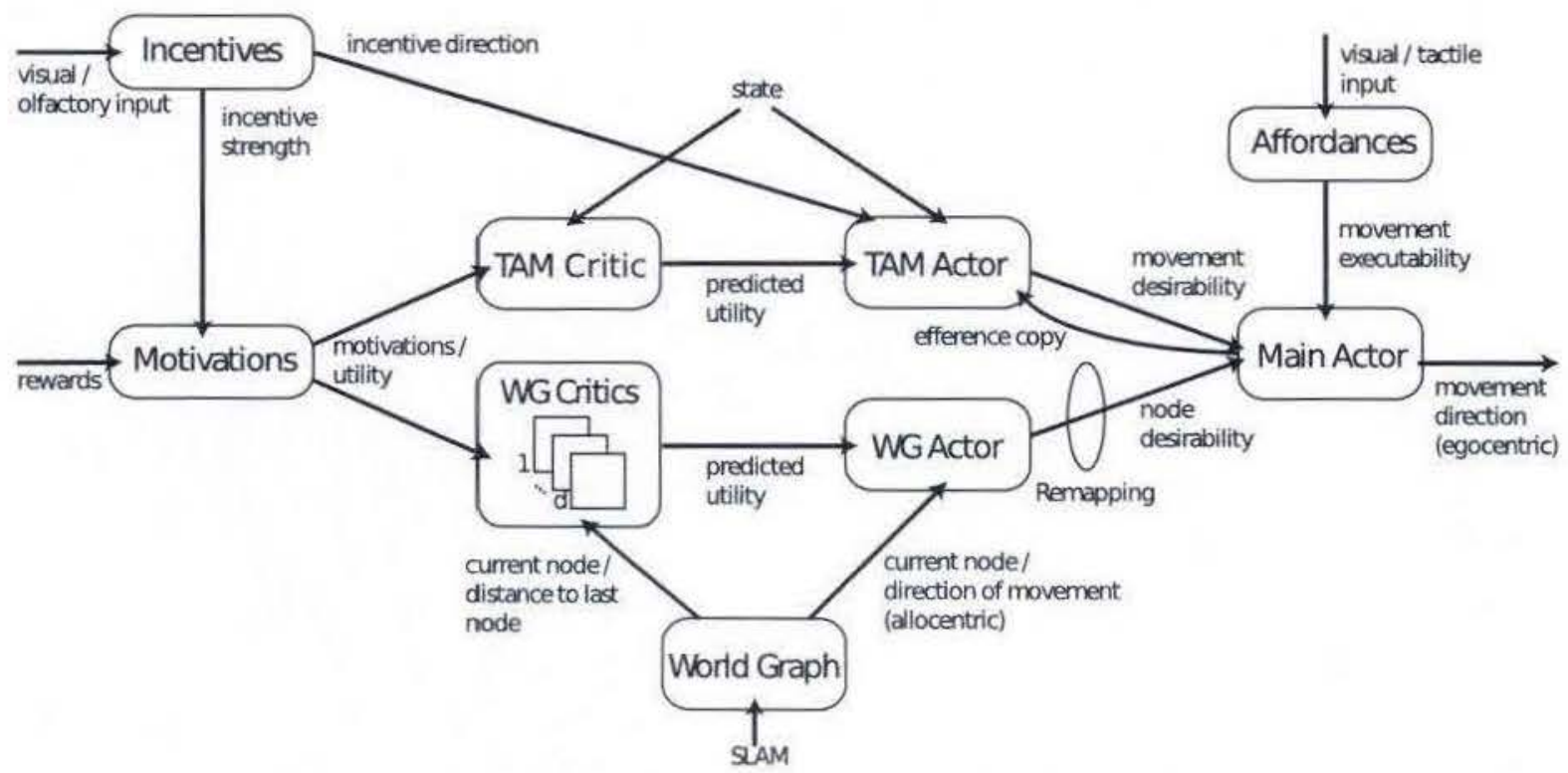

Figure 6. A functional view of the integrated Taxon Affordance Model-World Graph (TAM-WG) model. 
implementation have been discussed by Dominey and Arbib (1992).

The Premotor Cortex/Actor module multiplicatively combines the egocentric executability with the sum of the movement direction signal and the shifted node desirability signal to select the next movement direction. The input to the WTA process, $\mathbf{I}_{i n}$, now includes the shifted node desirability, ND:

$$
\begin{array}{r}
\mathbf{I}_{i n}(i, t)=\mathbf{E}(i, t)\left[\sum_{d}(\mathbf{S T}(d, i, t)+\mathbf{A D}(d, i, t)\right. \\
+\Phi(\mathbf{N D}(d, i, t), \theta))+\varepsilon]
\end{array}
$$

where $\varepsilon$ is uniformly distributed noise in the interval $[0,1]$.

\section{Results}

\section{I T-maze reversal}

O'Keefe (1983) sought to investigate the role of the hippocampus in navigation by testing control and fornixlesioned rats in a T-maze with food in one arm. After the rats reached a threshold level of performance on the T-maze, probe trials in an eight-arm maze were interspersed (Figure 7). Both control and lesioned rats were able to then reverse this association and learn a T-maze in which the reward is in the opposite arm, as it had been during training. However, whereas the control rats exhibited an abrupt shift in behavior from choosing the wrong arm to choosing the correct arm of the maze, lesioned rats show a steady shift in their directional preference (as gauged by the eight-arm probe trials).
We tested 10 instantiations of the model representing 10 rat subjects in a simulation of O'Keefe's experiment. Control rats were simulated using the full TAMWG model, while fornix-lesioned rats were simulating by severing the connection from the WG component to the Actor module so that only the TAM module had any influence on action decision-making. Each model instance was trained for 24 trials in the T-maze with food in the right arm (a $-90^{\circ}$ turn) with eight-arm maze probe trials every four trials. Performance was measured by calculating the percentage of time the model chose the correct arm in blocks of four trials. In the reversal condition, each model instance was run for 60 trials in the T-maze with food in the left arm (a $90^{\circ}$ turn), again with eight-arm maze probe trials every four trials.

Both the control and fornix-lesioned versions of the model were able to learn the initial $\mathrm{T}$-maze as well as the T-maze reversal. During initial training, the TAM and WG components developed policies that favored paths through the T-maze leading to the food in the right arm. Figure 8 shows traces from each layer (TAM on the left and WG on the right) for trials 1-10 (top to bottom) of the initial training period. The vertical axis of each plot provides a population code of orientation, with neuron 1 selective for the $\pi$ direction and neuron 200 selective for $-\pi$. The jump in representation seen around $3 \mathrm{~s}$ occurs when the model reaches the junction of the T-maze, representing a new node in the WG and a new recognizable situation or state for TAM. Note that the representation in TAM is in terms of egocentric direction of movement and is encoded as the model finds itself in a state corresponding to one of its recognizable situation. The WG encodes directions in
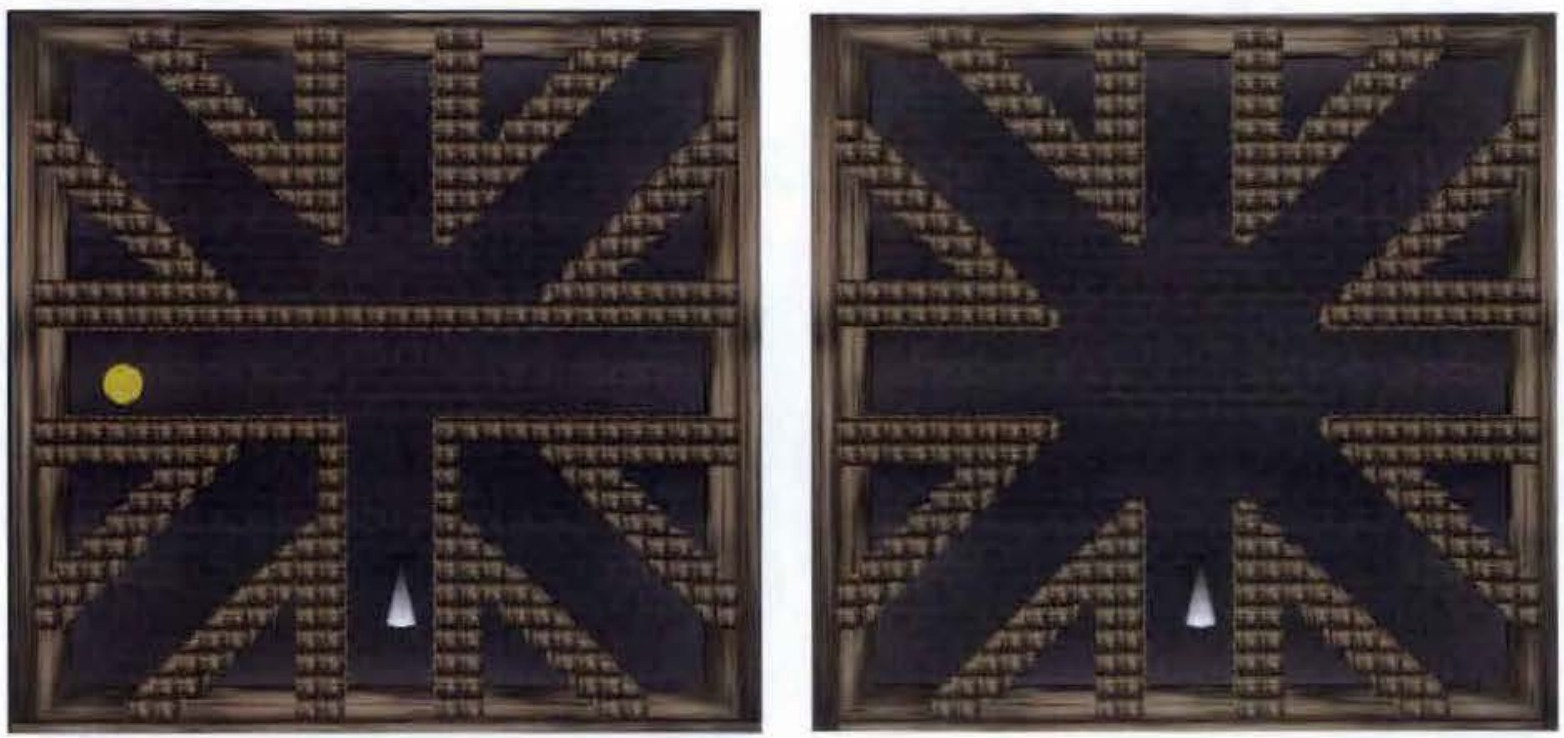

Figure 7. Left: the eight-arm maze with five arms blocked so that it becomes a T-maze. This maze has food in the left $\left(90^{\circ}\right)$ arm. Right: the same eight-arm maze unblocked for use in probe trials. 


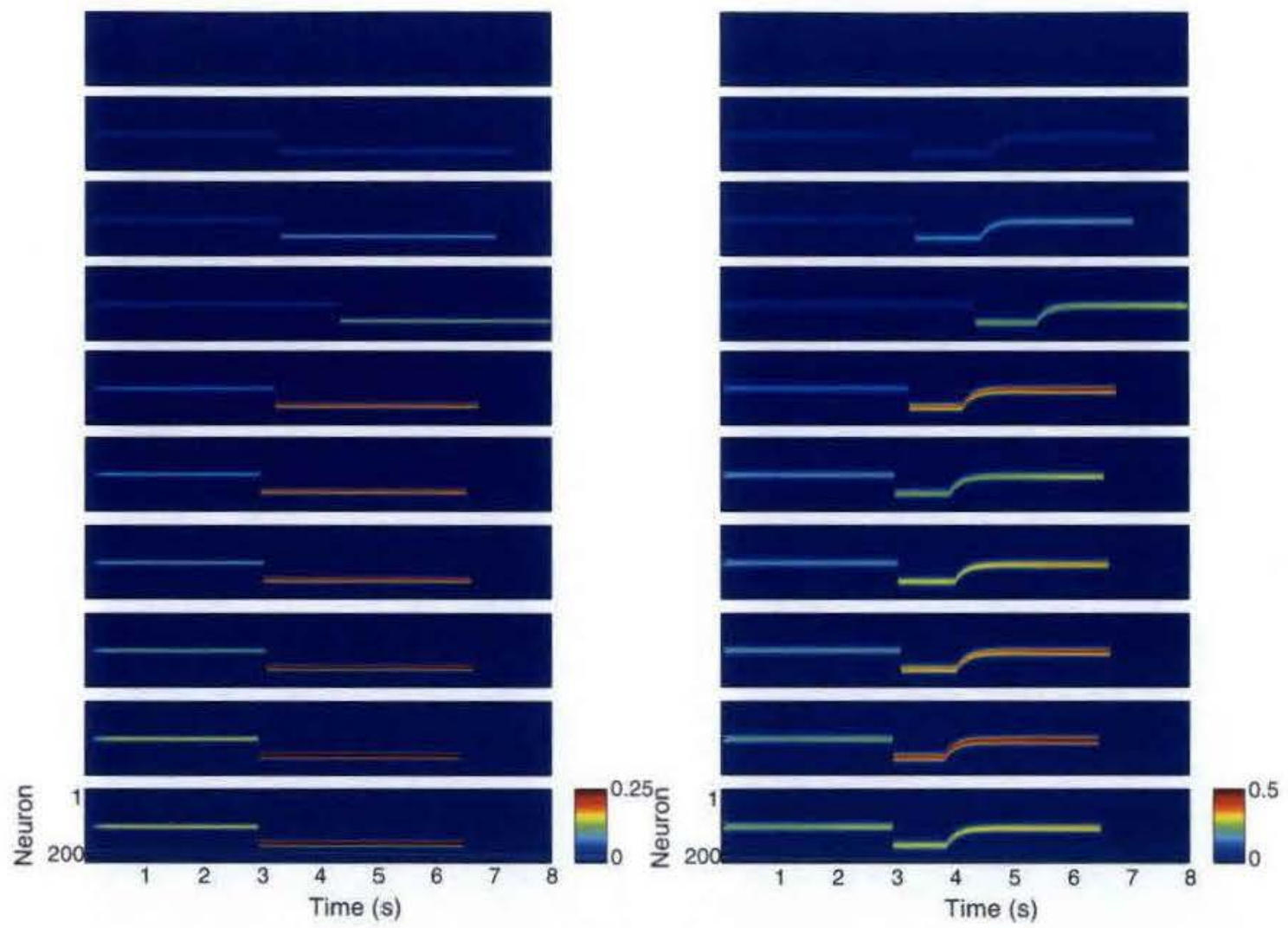

Figure 8. Left: Taxon Affordance Model (TAM) policy throughout trials in the control pretraining phase for trials $1-10$ (from top to bottom). The time is on the $x$-axis. The junction of the T-maze is reached at around $3 \mathrm{~s}$. The neuron number is on the $y$-axis, with neuron I selective for the direction and neuron 200 selective for -. Right: World Graph (WG) policy throughout trials in the control pretraining phase for trials $1-10$. The WG policy is rotated to be in an egocentric reference frame, thus the curved regions of activity show the policy updated as the model turns to face the direction it has chosen to move in.

terms of the directions of those allocentric landmarks that correspond to nodes in the current value of $W G(t)$, but rotated into an egocentric reference frame using the rat's current orientation. The curves in the WG representation in Figure 8 therefore occur after the model has selected a direction of travel and is rotating in order to then move in that direction. As the trials progress, the encodings of the forward direction in the first part of the T-maze and the right direction in the junction increase in strength. However, since the learning mechanism is based on temporal difference learning, the encoding strength increases in reverse from the junction to the start of the maze.

The fornix-lesioned version of the model was able to reproduce the behavior of the lesioned rats since the TAM component encodes the reward expectation as the center of mass over peaks in the raw policy. This representation is then multiplied by the affordance representation by the actor, resulting in a left or right decision in the T-maze, but revealing the underlying centerof-mass representation when more affordances are available in the eight-arm maze. The mean percentage of correct trials (over blocks of four) in the T-maze reversal condition for the fornix-lesioned model instances is shown on the left of Figure 9. The mean orientation decision for the same model instances during probe trials is shown in the right half of Figure 9. The first block of four trials in which a model instance performs at over $20 \%$ accuracy is labeled as trial 0 and used to align each instance's performance and orientation decision before averaging. At the start of the reversal condition, all model instances choose the right arm of the T-maze. The performance abruptly improves and increases to $100 \%$ as the trials progress, but the orientation decision in the probe trials smoothly moves from $-90^{\circ}$ to $90^{\circ}$, reflecting the march of activity in the TAM component as its center-of-mass representation encodes the increase in expected reward at $90^{\circ}$ and a decrease in expected reward at $-90^{\circ}$.

Without the simulated lesion, the full TAM-WG reproduced the behavior of the control rats in O'Keefe's experiment. The plots in Figure 10 show the mean performance of the lesioned model instances using the same conventions as Figure 9. Again the model performance in the reversed T-maze abruptly improves, but the orientation decision also abruptly 

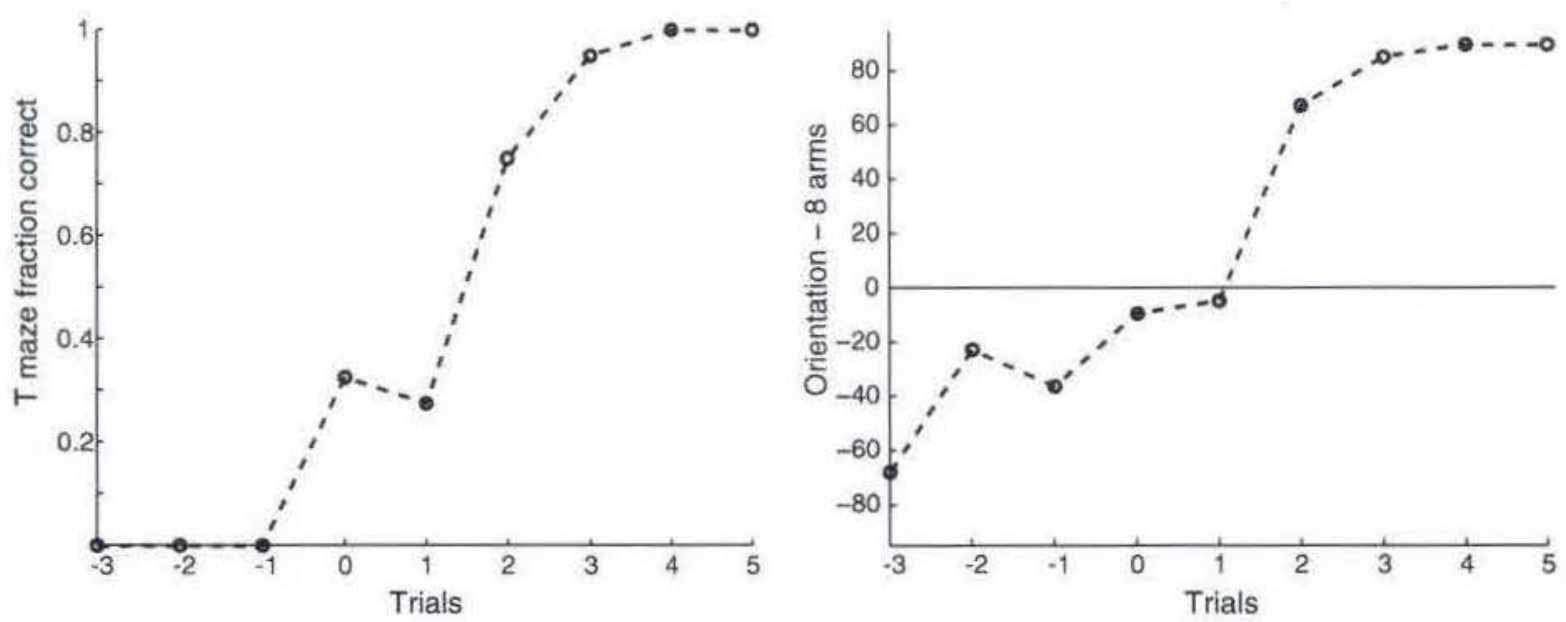

Figure 9. Left: mean fraction of correct trials for fornix-lesioned simulated rats in the T-maze reversal task. The plot was obtained by averaging over the performance of all rats after aligning to the trial in which they reached at least $20 \%$ correct (marked trial 0 ). Each rat's performance was computing by taking the percentage of correct trials in blocks of four. Right: mean orientation decision for fornix-lesioned rats in the eight-arm maze during probe trials of the T-maze reversal task.
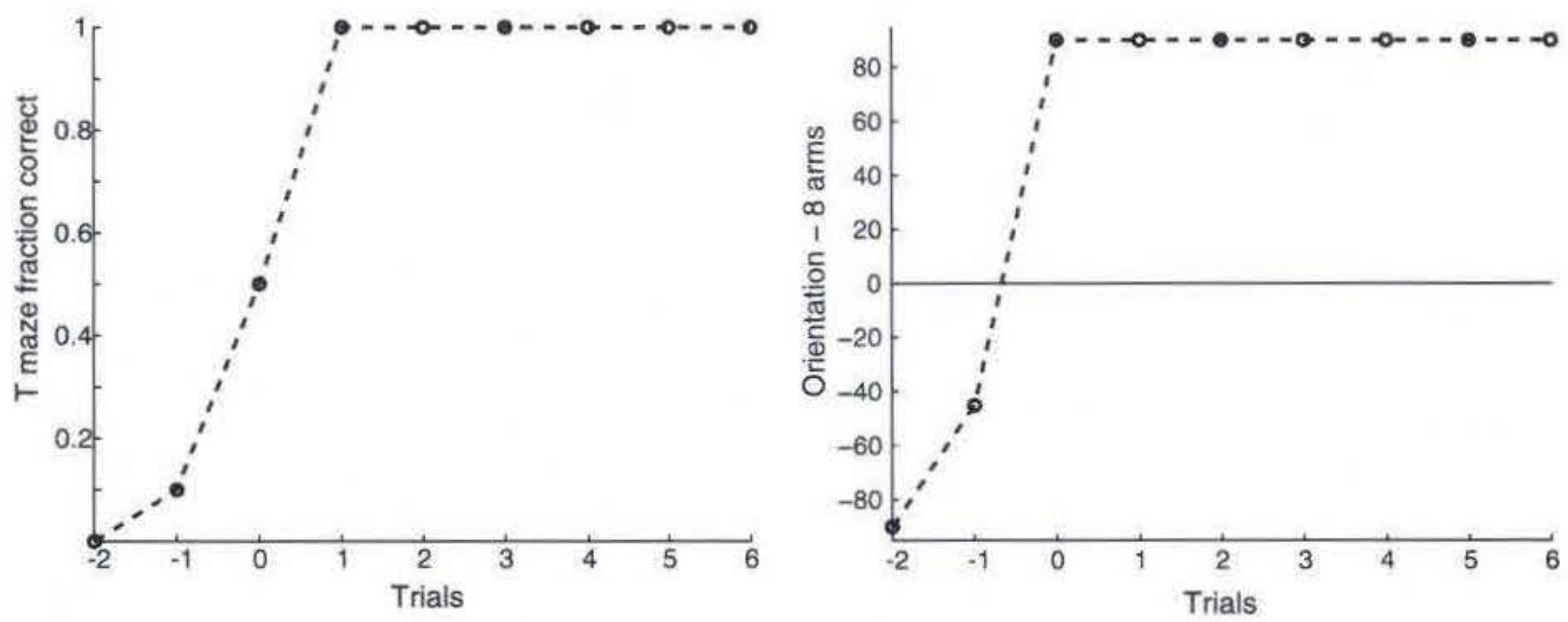

Figure 10. Left: mean fraction of correct trials for control simulated rats in T-maze reversal task. The plot was obcained as that in Figure 9. Right: mean orientation decision for fornix-lesioned rats in the eight-arm maze during probe trials of the T-maze reversal task.

shifts from $-90^{\circ}$ to $90^{\circ}$. Unlike the TAM module, the WG does not represent directions using a center-offmass since it stores the relative orientation of different nodes in the graph. In trial 1 , the WG layer favors a $-90^{\circ}$ turn, but this preference is quickly reduced and a bias towards the $90^{\circ}$ turn is evident by trial 6 before the TAM layer has even started to shift its representation.

\subsection{Automatic detour behavior in the face of obstacles}

When an obstacle is present in a previously learned maze, the interaction of the WG and TAM components automatically yields detour behavior. In order to demonstrate this feature, we trained the model on a maze containing multiple routes from the starting position to the food, each of a different length (Figure 11A). The model was trained on the maze for 75 trials, each starting in a random part of the maze. The first time the model entered the novel maze, the WG contained a single node representing the starting location. Nearby landmarks at locations not yet represented in the graph were added as nodes, with edges connecting to them from the current node if an affordance was perceived in that direction. As the model traversed these edges and obtained the food, the desirability of each node was modified according to the learning 

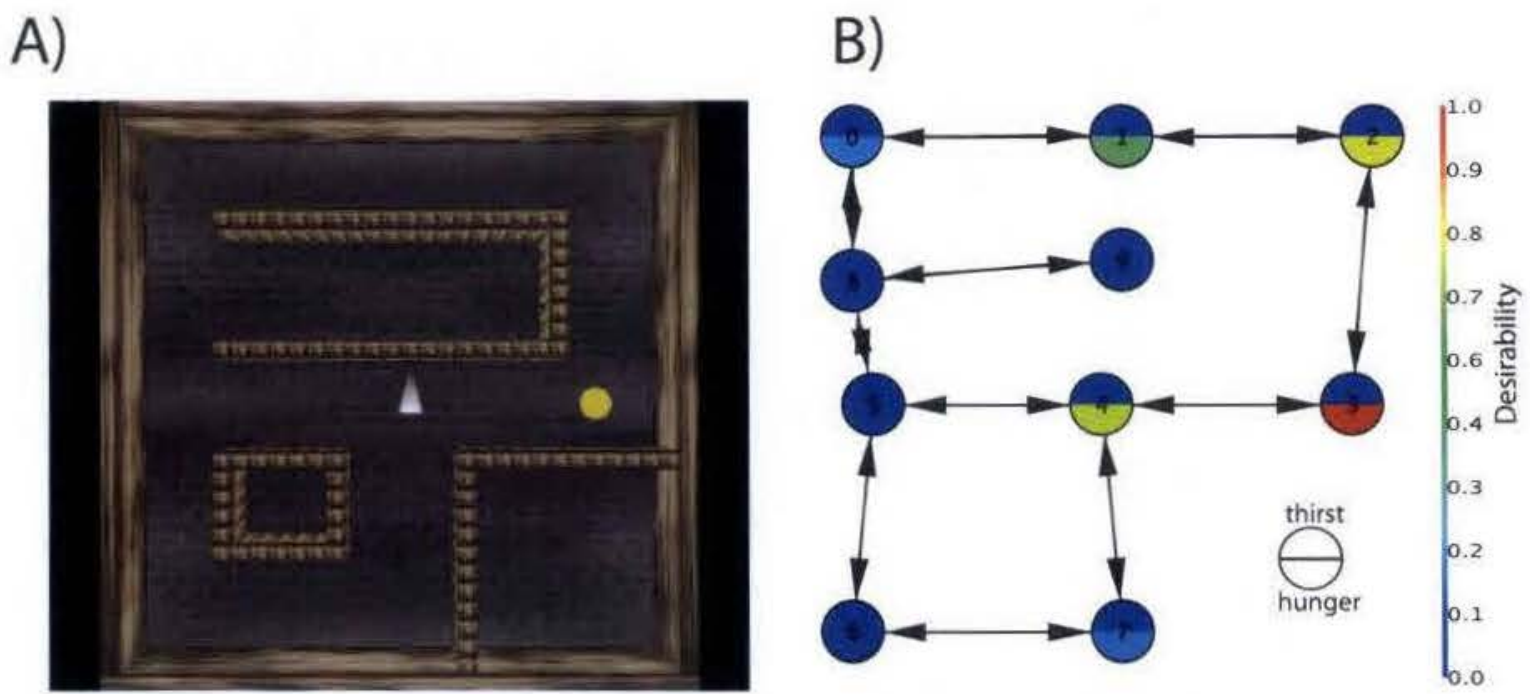

C)

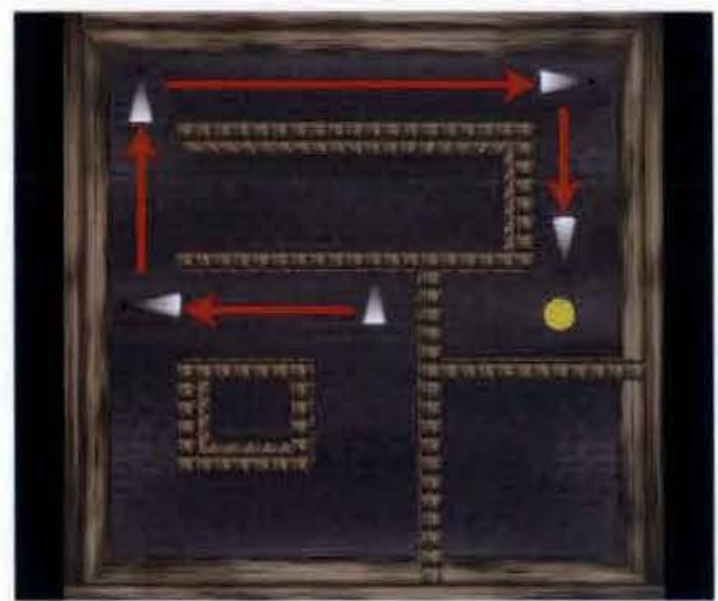

D)

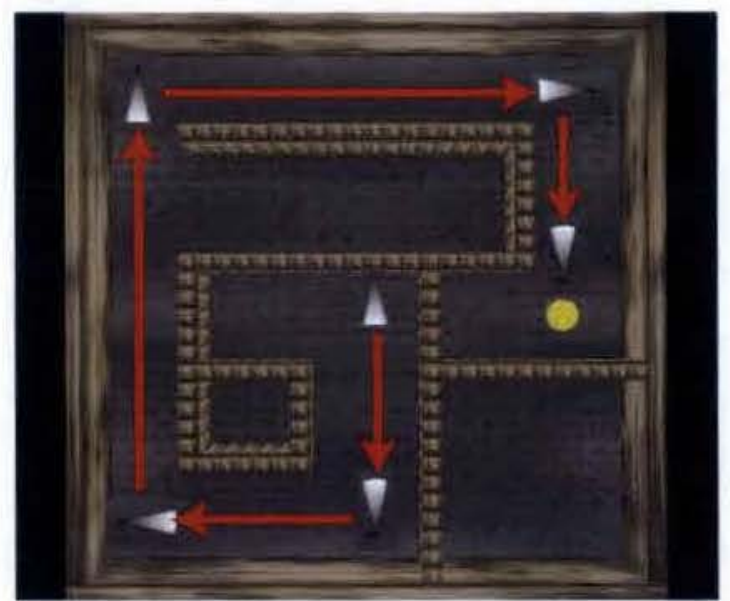

Figure II. (A) The maze used to test detour behavior. (B) The World Graph that resulted from training. The desirability of each node is represented by its color with the top half represent desirability in the thirst dimension and the bottom color. This maze contained food and no water therefore the desirability in the thirst dimension is zero for each node. (C) Model behavior when the shortest path to the food is blocked. (D) Model behavior when both the shortest and second shortest paths are blocked.

algorithm described above. This resulted in a complete WG with node desirabilities according to each node's distance to the food (Figure 11B).

After training the model on the maze, obstacles were introduced, which blocked one or more routes through the maze. When the model finds itself adjacent to an obstacle, the node desirability and action desirability signals still encode the preferred direction of movement even though it may be blocked. However, the executability signal from the parietal cortex no longer encodes the blocked direction as a locomotive affordance. The multiplicative combination of the executability and desirability signals in the computation of priority ensures that only unobstructed directions are selected for movement. This enables the model to traverse alternate paths through the maze by moving in directions with the highest priority (Figures $11 \mathrm{C}$ and D).

\subsection{Spatial difference learning and the dual (at least) characterization of space}

In order to demonstrate the interaction of the locometric and cognitive map spaces, we tested the model in a linear maze with four nodes and food in the farthest node (Figure 12, middle). (The model bears some similarity to that of Voicu \& Schmajuk, 2000, but they fail to distinguish the locometric map from the more abstract map of the kind provided by WG.) The model was trained for 20 trials on the linear maze, which contained food in one end. One the 21 st trial, a shock was 


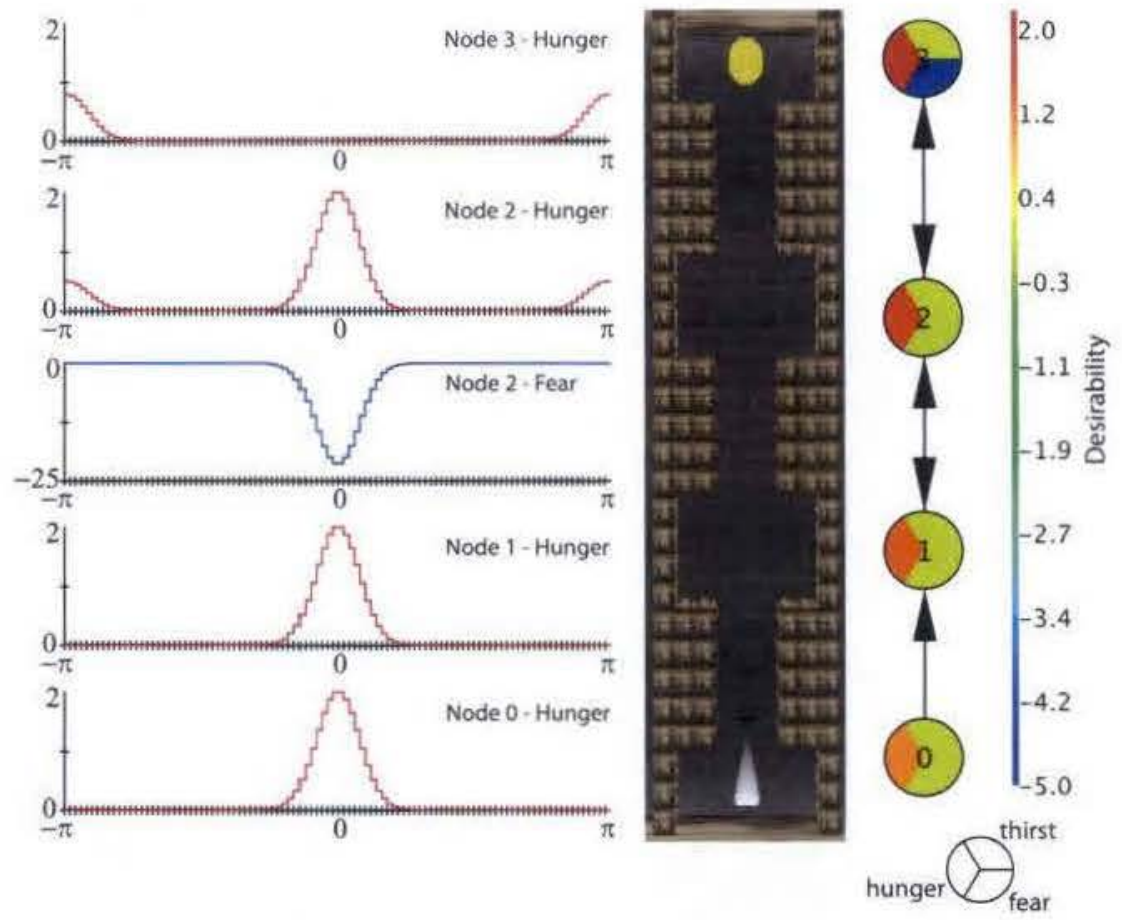

Figure 12. Left: the World Graph (WG) actor's raw policy for the hunger and fear drives for each node in the linear maze. Due to heavy discounting in the WG critic for the fear drive, only node 2 has a non-zero fear policy. Middle: an overview of the linear maze. Right: the WG critic's node value estimates.

given at the same time as the food was acquired. The model was then tested in the same maze. In this simulation, the fear drive was given a small, tonic input equal to 1.25 . Prior to receiving the shock, the WG critic learned an estimate of each node's value with respect to the hunger drive, resulting in a gradient of desirability from the start of the maze, reaching its peak at the end where the food was located. Upon receiving the shock, the WG critic quickly adjusted its estimate of the desirability of node 3 (corresponding to the end of the maze where the food and shock were located) with respect to the fear drives to -5 (Figure 12, right). Based on the output of the WG critic, the WG actor learned a policy that consisted of an attractive field in the direction of node 3 and a repulsive field in node 2 away from node 3 (Figure 12, left).

We further examined this phenomenon by taking a closer look at the activity in the WG layer just before and just after its policy shifts from positive to negative as the model approaches the node containing the food and where it had received a shock (Figure 13). When the WG actor computes the node desirability signal, the effective policy is obtained by weighting each drive dimension of the raw policy by the drive value, biasing by the current distance to the node encoded by each peak in the population, and summing over all drives. The distance bias depends on the value of the discount factor for each drive, $\kappa_{d}$ (see Materials and Methods,

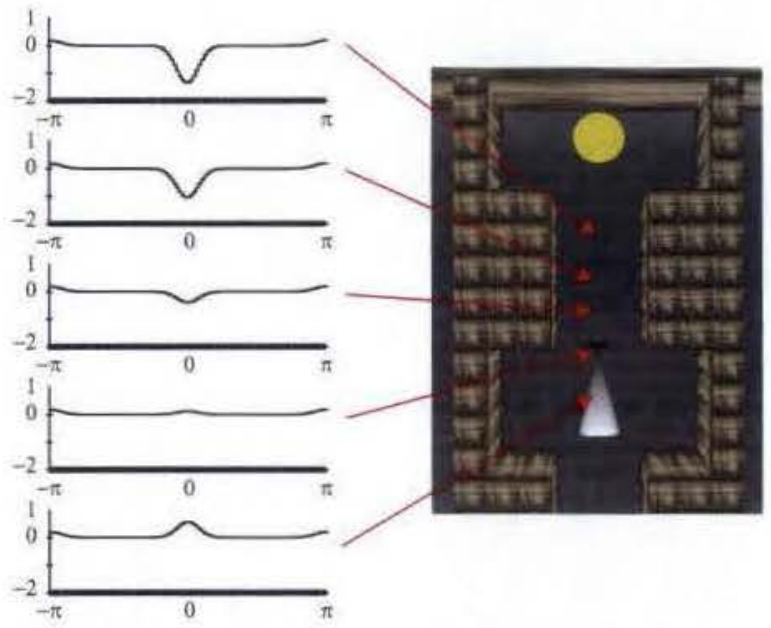

Figure 13. The World Graph (WG) actor's effective policy after weighting by drive level, biasing by distance to node 3 , and summing over all drives, during various points on the path from node 2 to node 3 .

the WG Model). The hunger discount factor is much greater than the fear discount factor (see Appendix, Table 3), meaning that the effective value of hunger policies does not decrease much with distance, whereas the effective value of fear policies falls off rapidly with distance to the next node. Therefore as the model approaches node 3 from 2 , the effective policy is 
initially solely determined by the hunger policy but is gradually dominated by the fear policy (Figure 13). When this happens, the activation in the actor layer representing the direction the model is moving in is decreased, and the neurons representing the reverse direction begin to fire. This interrupts the motor controller, sets a new target location, which causes the model to turn around and move away from the food and the location of the remembered shock.

The point here was that the appetitive attraction of food as a function of locometric space in the path from node 2 to 3 remains high at node 2 , whereas the aversive repulsion of food in is near 0 at node 2 . There is thus a crossover point $\mathrm{X}$ not in the cognitive map - as a result, the rat runs from node 2 towards node 3 , but on passing $\mathrm{X}$ turns tail and runs back towards node 2, passing $\mathrm{X}$ again and reaching a point where hunger overcomes fear ... and thus ends up oscillating between the nodes 2 and 3 .

\section{Discussion}

The TAM-WG model was redeveloped within the new ACQ framework (Bonaiuto \& Arbib, 2010). What the original TAM-WG model called reward expectation we call action and node desirability, and affordances are incorporated in ACQ's notion of executability. The main difference is that in this model the actor multiplies the executability and desirability signals whereas in the original TAM-WG model their counterparts are added. Adding these signals results in a situation where even impossible movements are input into the WTA network and the model relies on the fact that the lack of executability signal in these directions makes their priority lower than those of possible movements. However, the input is noisy and this situation could result in impossible movement directions being selected for action. Multiplication allows more effective gating of desirability by greatly reducing a direction's priority when its executability is low. This implementation remains biologically plausible since it has been shown that single neurons can multiplicatively combine inputs (Mel, 1993) and that the multiplicative effect of gain modulation can be achieved in population codes (Salinas \& Abbott, 1996).

A difference between this TAM-WG and the plain ACQ model is the addition of the WG module. In this model, the WG module exerts a sort of higher-level desirability influence on action selection. The output is a broad bias over all directions in the range of the direction of desirable adjacent nodes. The actor combines the coarse node desirability bias with the more accurate executability, incentive, and action desirability signals. This development might be used to extend ACQ to handle hierarchically organized actions. By analogy with the WG module, the Prefrontal Cortex might contain a directed graph of goal states, with each edge exerting a broad desirability bias on actions that might achieve that goal state from the current environmental state. In the specialized domain of spatial navigation, the node desirability bias can be computed using the orientation of the edge connecting two nodes. The situation is more complex for actions in general because the actions that will achieve a goal state are not known a priori and therefore the desirability bias must also be learned.

\section{Funding}

Grant sponsor: National Science Foundation Program in Perception, Action, and Cognition. Grant number: BCS0924674 (Michael A. Arbib, Principal Investigator)

\section{References}

Amari, S., \& Arbib, M. (1977). Competition and cooperation in neural nets. In J. Metzler (Ed.), Systems neuroscience (pp. 119-166). New York, NY: Academic Press.

Arbib, M. A. (1997a). From visual affordances in monkey parietal cortex to hippocampo-parietal interactions underlying rat navigation. Philosophical Transactions of the Royal Society B: Biological Sciences, 352, 1429-1436.

Arbib, M. A. (1997b). Modeling visuomotor transformations. In M. Jeannerod (Ed.), Handbook of neuropsychology, Volume 11. Section 16: Action and cognition (pp. 65-90). Amsterdam, The Netherlands: Elsevier.

Arbib, M. A., \& Lieblich, I. (1977). Motivational learning of spatial behavior. In J. Metzler (Ed.), Systems neuroscience (pp. 221-239). New York, NY: Academic Press.

Bonaiuto, J. B., \& Arbib, M. A. (2010). Extending the mirror neuron system model, II: What did I just do? A new role for mirror neurons. Biological Cybernetics, 102, 341-359.

Borgland, S. L., Storm, E., \& Bonci, A. (2008). Orexin B/ hypocretin 2 increases glutamatergic transmission to ventral tegmental area neurons. European Journal of Neuroscience, $28,1545-1556$.

Calton, J. L., \& Taube, J. S. (2009). Where am I and how will I get there from here? A role for posterior parietal cortex in the integration of spatial information and route planning. Neurobiology of Learning and Memory, 91, 186-196.

Colby, C. L. (1998), Action-oriented spatial reference frames in cortex. Neuron, 20, 15-24.

Dominey, P. F., \& Arbib, M. A. (1992). A cortico-subcortical model for generation of spatially accurate sequential saccades. Cerebral Cortex, 2, 153-175.

Erlhagen, W., \& Schoner, G. (2002). Dynamic field theory of movement preparation. Psychological Review, 109, 545572.

Gibson, J. J. (1955). The optical expansion-pattern in aerial location. American Journal of Psychology, 68, $480-484$.

Goldman, A. (2006). Simulating minds: The philosophy, psychology, and neuroscience of mindreading. Oxford, UK/ New York, NY: Oxford University Press.

Guazzelli, A., Corbacho, F. J., Bota, M., \& Arbib, M. A. (1998). Affordances, motivation, and the World Graph Theory. Adaptive Behavior, 6, 435 471.

Guazzelli, A., Bota, M., \& Arbib, M.A. (2001). Competitive Hebbian learning and the hippocampal place cell system: 
modeling the interaction of visual and path integration cues. Hippocampus, $1 /(3)$ : 216-239.

Lieblich, I., \& Arbib, M. A. (1982). Multiple representations of space underlying behavior. The Behavioral and Brain Sciences, 5, 627-659.

McNaughton, B. L., Leonard, B., \& Chen, L. (1989). Cortical-hippocampal interactions and cognitive mapping: $\mathrm{A}$ hypothesis based on reintegration of the parietal and inferotemporal pathways for visual processing. Psychobiology. $17,236-246$.

McNaughton, B. L., Mizumori, S. J., Barnes, C. A., Leonard, B. J., Marquis, M., \& Green, E. J. (1994). Cortical representation of motion during unrestrained spatial navigation in the rat. Cerebral Cortex, 4, 27-39.

Mel, B. (1993). Synaptic integration in an excitable dendritic tree. Journal of Neurophysiology, 70, 1086-1101.

Milford, M., Schulz, R., Prasser, D., Wyeth, G., \& Wiles, J. (2007). Learning spatial concepts from RatSLAM representations. Robotics and Autonomous Systems-From Sensors to Human Spatial Concepts, 55, $403-410$.

Milford, M., \& Wyeth, G. (2010). Persistent navigation and mapping using a biologically inspired SLAM system. The International Journal of Robotics Research, 29, 1131-1153.

Milford, M., Wyeth, G., \& Prasser, D. (2004). IEEE International Conference on Robotics and Automation, New Orleans, USA (pp. 403-08). IEEE.

Nitz, D. (2009). Parietal cortex, navigation, and the construction of arbitrary reference frames for spatial information. Neurobiology of Learning and Memory, 91, 179-185.

O'Keefe J. (1983). Spatial memory within and without the hippocampal system. In W. Seifert (Ed.), Neurobiology of the Hippocampus (pp. 375-403). New York, NY: Academic Press.

O'Keefe, J., \& Dostrovsky, J. (1971). The hippocampus as a spatial map: Preliminary evidence from unit activity in the freely moving rat. Brain Research, 34, 171-175.

O'Keefe, J., \& Nadel, L. (1978). The hippocampus as a cognitive map. Oxford, UK: Oxford University Press.

Pan, W. X., Schmidt, R., Wickens, J. R., \& Hyland, B. I. (2005). Dopamine cells respond to predicted events during classical conditioning: Evidence for eligibility traces in the reward-learning network. Journal of Neuroscience, 25 , $6235-6242$.

Sakurai, T. (2006). Roles of orexins and orexin receptors in central regulation of feeding behavior and energy homeos- tasis. CNS \& Neurological Disorders - Drug Targets, 5 , 313-325.

Salinas, E., Abbott, L. (1996), A model of multiplicative neural responses in parietal cortex. Proceedings of the National Academy of Sciences of the USA, 93, 1195611961.

Save, E., Paz-Villagran, V., Alexinsky, T., \& Poucet, B. (2005). Functional interaction between the associative parietal cortex and hippocampal place cell firing in the rat. European Journal of Neuroscience, 21, 522-530.

Singh, S. P., \& Sutton, R. S. (1996). Reinforcement learning with replacing eligibility traces. Machine Learning, 22, 123-158.

Srinivasan, M. V., Zhang, S., Altwein, M., \& Tautz, J. (2000). Honeybee navigation: Nature and calibration of the "odometer". Science, 287, 851-853.

Sutton, R. S. (1984). Temporal credit assignment in reinforcement learning. Doctoral dissertation, Computer and Information Science, University of Massachusetts.

Sutton, R. S., \& Barto, A. G. (1998). Reinforcement learning: An introduction. Cambridge, MA: The MIT Press.

Swanson, L., \& Mogenson, G. (1981). Neural mechanisms for the functional coupling of automatic endocrine and somatomotor responses in adaptive behavior. Brain Research Reviews, 3, 1-34.

Swanson, L. W. (2000). Cerebral hemisphere regulation of motivated behavior. Brain Research, 886, 113-164.

Swanson, L. W. (2005). Anatomy of the soul as reflected in the cerebral hemispheres: Neural circuits underlying voluntary control of basic motivated behaviors. Journal of Comparative Neurology, 493, 122-131.

Vittoz, N. M., Berridge, C. W. (2006). Hypocretin/orexin selectively increases dopamine efflux within the prefrontal cortex: Involvement of the ventral tegmental area. Neuropsychopharmacology, 31, 384-395.

Voicu, H., \& Schmajuk, N. (2000). Exploration, navigation and cognitive mapping. Adaptive Behavior, 8, 207-223.

Weitzenfeld, A., Arbib, M. A., \& Alexander, A. (2002). The neural simulation language: A system for brain modeling. Cambridge, MA: The MIT Press.

Wu, S., Amari, S., \& Nakahara, H. (2002). Population coding and decoding in a neural field: A computational study. Neural Computation, 14, 999-1026.

\section{About the Authors}
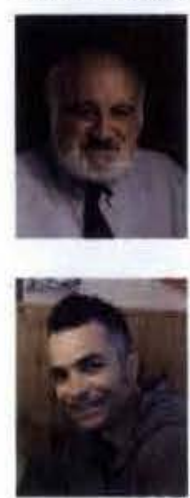

Michael Arbib is a university professor and director of the USC brain project at the University of Southern California and a board member of the Academy of Neuroscience for Architecture. His first book was Brains, Machines and Mathematics (McGraw-Hill, 1964) and his fortieth book is How the Brain Got Language: The Mirror System Hypothesis (Oxford University Press, 2012).

James Bonaiuto obtained his Bachelor's degree in computer science from Drexel University in 2004. He received a Ph.D. in neuroscience from the University of Southern California as a student of Michael Arbib in 2010. He has published several papers on computational models of the mirror system and is currently a post-doctoral fellow at the California Institute of Technology under the supervision of Richard Andersen. 


\section{Appendix}

Table I. Motivational system parameters

\begin{tabular}{|c|c|c|c|}
\hline Parameter & Description & Value & Justification \\
\hline$\alpha_{\text {hunger }}$ & Hunger gain parameter & 0.000025 & $\begin{array}{l}\text { Small enough that hunger does not significantly increase of the } \\
\text { course of a } 2-5 \text { simulation }\end{array}$ \\
\hline$\alpha_{\text {thirst }}$ & Thirst gain parameter & 0.000025 & $\begin{array}{l}\text { Small enough that thirst does not significantly increase of the } \\
\text { course of a 2-s simulation }\end{array}$ \\
\hline$\alpha_{\text {fear }}$ & Fear gain parameter & 0.1 & $\begin{array}{l}\text { Large enough so fear decreases quickly after the cessation of } \\
\text { aversive stimuli }\end{array}$ \\
\hline$d_{\text {hunger }}$ & Minimum hunger level & 0 & Chosen for computational convenience \\
\hline dhungerines & Maximum hunger level & 2 & $\begin{array}{l}\text { Must be smaller than } d_{\text {fear }} \text { for fear to overcome hunger as a } \\
\text { node with aversive stimuli is approached }\end{array}$ \\
\hline$d_{\text {thinst }}$ & Minimum thirst level & 0 & Chosen for computational convenience \\
\hline$d_{\text {thirst }}$ & Maximum thirst level & 2 & Set equal to dhunger \\
\hline$d_{\text {fearmen }}$ & Minimum fear level & 0 & Chosen for computational convenience \\
\hline$d_{\text {fear }_{\max }}$ & Maximum fear level & 5 & $\begin{array}{l}\text { Large enough to overcome the hunger and thirst drives when } \\
\text { approaching aversive stimuli }\end{array}$ \\
\hline$c_{\text {hunger }}$ & $\begin{array}{l}\text { Hunger reduction for one bite } \\
\text { of food }\end{array}$ & 0.2 & $\begin{array}{l}\text { Set to } \frac{d_{\text {hurererax }}}{10} \text {, determines the magnitude of food reward for one } \\
\text { time step }\end{array}$ \\
\hline$c_{\text {thirst }}$ & $\begin{array}{l}\text { Thirst reduction for one sip of } \\
\text { water }\end{array}$ & 0.2 & $\begin{array}{l}\text { Set to } \frac{d \text { minsan }}{10} \text {, determines the magnitude of fear reward for one } \\
\text { time step }\end{array}$ \\
\hline$c_{\text {fear }}$ & Fear reduction for one shock & -5 & $\begin{array}{l}\text { Set to }-d_{\text {feorma }} \text { so that fear drive is driven to its maximal level by } \\
\text { one shock }\end{array}$ \\
\hline ST(hunger) & $\begin{array}{l}\text { Strength of incentive stimuli for } \\
\text { smell/sight of food }\end{array}$ & 0.1 & Set to $\frac{C_{\text {nurer }}}{2}$, must be less than chunger $_{\text {for consistency }}$ \\
\hline ST(thirst) & $\begin{array}{l}\text { Strength of incentive stimuli for } \\
\text { sight/sound of water }\end{array}$ & 0.1 & Set to $\frac{c_{\text {sing }}}{2}$, must be less than $c_{\text {thirst }}$ for consistency \\
\hline
\end{tabular}

Table 2. Taxon Affordance Model (TAM) model parameters

\begin{tabular}{|c|c|c|c|}
\hline Parameter & Description & Value & Justification \\
\hline$\gamma$ & Discount factor & 0.5 & $\begin{array}{l}\text { Large enough to propagate hunger rewards three or four nodes } \\
\text { back }\end{array}$ \\
\hline$\beta_{\text {TAM }}$ & Critic learning rate & 0.1 & $\begin{array}{l}\text { Determines rate of state value changes - the model becomes } \\
\text { unstable when this value is too large. Set lower than } \beta_{W G} \text { to } \\
\text { ensure that the influence of the WG dominates action selection. }\end{array}$ \\
\hline$\eta_{\text {TAM }}$ & Actor learning rate & 0.01 & $\begin{array}{l}\text { Determines the rate of node policy changes - the model } \\
\text { becomes unstable when this value is larger than } \beta_{\text {TAM }}\end{array}$ \\
\hline
\end{tabular}

Table 3. World Graph (WG) model parameters

\begin{tabular}{|c|c|c|c|}
\hline Parameter & Description & Value & Justification \\
\hline Yhunger & Hunger discount factor & 0.9 & Large enough to propagate hunger rewards back to early nodes \\
\hline$\gamma_{\text {thirst }}$ & Thirst discount factor & 0.9 & Large enough to propagate thirst rewards back to early nodes \\
\hline$\gamma_{\text {fear }}$ & Fear discount factor & 0.01 & $\begin{array}{l}\text { Small enough to not propagate aversive stimuli more than one or } \\
\text { two nodes back }\end{array}$ \\
\hline$\beta_{W G}$ & Critic learning rate & 0.3 & $\begin{array}{l}\text { Determines rate of node value changes - the model becomes } \\
\text { unstable when this value is too large }\end{array}$ \\
\hline$\eta_{\text {We }}$ & Actor learning rate & 0.2 & $\begin{array}{l}\text { Determines the rate of node policy changes - the model } \\
\text { becomes unstable when this value is larger than } \beta_{W G}\end{array}$ \\
\hline$\kappa_{\text {hunger }}$ & Hunger edge length discount factor & 100 & $\begin{array}{l}\text { Large enough that hunger policies are effective over large } \\
\text { distances }\end{array}$ \\
\hline$K_{\text {thirst }}$ & Thirst edge length discount factor & 100 & Small enough that thirst policies are effective over large distances \\
\hline$\kappa_{\text {fear }}$ & Fear edge length discount factor & 3.5 & $\begin{array}{l}\text { Approximately one quarter of the edge length in the linear maze } \\
\text { so that fear overwhelms hunger at the halfway point }\end{array}$ \\
\hline
\end{tabular}

